\title{
CHEMICAL AND SENSORY CHARACTERISTICS OF SVRLJIG WHITE CHEESE ${ }^{1}$
}

\author{
O. Maćej, Snežana Jovanović, M. Barać, Sanja Seratlić, Tanja Vučić ${ }^{2}$
}

Contents: The aim of this paper was to signify the main characteristics of Svrljig white cheese in brine. Svrljig white cheese is producing from ewe's, cow's or mixed milk and differs with high fat in dry matter content (47.44\% and54.04\%), which ranks it in group of full fat cheeses. According to water content in solids non fat, this cheese belongs to group of soft cheeses $(69.40 \%$ and $67.99 \%)$. Cheese had good sensor characteristics such as taste, aroma and color were typical for cheeses made from cow's and ewe's milk. High acidity and low $\mathrm{pH}$ are characteristics for white cheese in brine group, in which Svrljig cheese belongs.

Key words: white cheeses in brine, ewe's milk, cow's milk

\section{Introduction}

Milk production in mountain area is related to a production of autochthonous milk products beside all. The production of these cheeses in mountain area of Serbia territory is running in domestic households and cottages on mountains by summer (serb. bačije, katuni, stanovi), which is the characteristic of trade milk production. Autochthonous milk production can give quite contribution to animal husbandry development and geographical regions animation. Besides, autochthonous milk products may be a good basis for assortment expansion of quality, original cheeses.

White cheeses in brine are large group of cheeses world wide. The characteristic of these cheeses is ripening in brine, in which not only ripening occurs, but cheese preservation as well. The general characteristics of these cheeses are higher acidity, sharp salty flavour as well as compressed, monolith and fragile consistency.

The autochthonous production of Svrljig white cheese in brine imply raw ewe's milk usage, while the production of white cheese in brine in type of Svrljig white cheese imply mixed cow's and ewe's milk usage. The ewe's white cheese in brine production is characteristic, especially in period of April till the end of October, during ewe's pasture.

\section{Materials and methods}

For chemical composition of Svrljig white cheese in brine determination 13 samples ( 7 cow's cheese samples and 6 ewe's cheese samples) has been taken. The following analyses were made:

- Determination of total solids by standard drying method at $102 \pm 2^{\circ} \mathrm{C}$ (Carić et al., 2000);

- Determination of milk fat according to van Gulik method (Carić et al., 2000);

- Determination of total nitrogen content with Kjeltec system by Kjeldahl method (Carić et al., 2000);

- Determination of soluble nitrogen content according to Van Slyke and Hart method (Pejić and Đorđević, 1963);

- Determination of primary and secondary nitrogen content according to Đorđević modified method (Pejić and Đorđević, 1963);

- Determination of salt according to Mohr (Carić et al., 2000);

- Determination of ash content by standard method at $550^{\circ} \mathrm{C}$ (Carić et al., 2000);

- Determination of titratable acidity according to Soxhlet-Henkel method (Carić et al., 2000);

- Determination of $\mathrm{pH}$ with $\mathrm{pH}-$ meter Sentron 1001

Determination of Svrljig white cheese in brine sensor characteristics was performed with scoring rating method - 5 level scale (Radovanović and Popov-Raljić, 2001). The commission was composed of 5 experts from the field of milk technology. Sensory evaluation is given as mean value and as a percent of total

1 Original scientific paper - originalni naučni rad

2 dr Ognjen Maćej, PhD, dr Snežana Jovanović, doc, dr Miroljub Barać, doc, Sanja Seratlić, Bsc, teaching assistant, Tanja Vučić, Bsc., assistant. Faculty of Agriculture, Belgrade 
possible quality. For determination of percent of maximal possible quality the following coefficients of importance are used: general appearance -2 ; cut -3 ; colour -2 ; aroma -2 ; consistency -3 and taste -8 .

\section{Results and discussion}

The Svrljig white cheese in brine production is running in domestic households or cottages in the mountain by summer. According to Dozet et al., 1996. in region of Niš, where Svrljig belongs, white cheeses in brine are most produced among the autochthonous dairy products. They carry different names as Beli, Sirenje, Velija, Kriška etc.

There are varieties in production process, but they have no relevant influence on cheese characteristics, principally the colour and cheese curd, which is closed and has a small number of holes and milky acid taste due to ripening in brine or whey.

\section{Chemical composition of Svrljig white cheese in brine}

Chemical composition of Svrljig white cheese in brine is given in table 1.

Table 1. Chemical composition of Svrljig white cheese

\begin{tabular}{|c|c|c|c|c|c|}
\hline \multirow{2}{*}{ Investigated parameters } & \multicolumn{5}{|c|}{ Statistical parameters } \\
\hline & $\min$. & $\max$. & $X(n=7)$ & $\mathrm{S}_{\mathrm{d}}$ & $\mathrm{Cv}(\%)$ \\
\hline \multicolumn{6}{|c|}{ CHEESE MADE OF COW'S MILK } \\
\hline TS (\%) & 42.10 & 51.40 & 46.75 & 3.3893 & 7.25 \\
\hline Moisture (\%) & 48.60 & 57.90 & 53.25 & 3.3893 & 6.36 \\
\hline MFFB (\%) & 61.90 & 76.89 & 69.40 & 5.1501 & 7.42 \\
\hline Fat $(\%)$ & 16.00 & 27.50 & 21.75 & 4.7845 & 22.00 \\
\hline FTS (\%) & 33.33 & 61.55 & 47.44 & 9.9162 & 20.90 \\
\hline Total nitrogen $(\%)$ & 1.9053 & 2.3862 & 2.1458 & 0.1562 & 7.28 \\
\hline Proteins $(\%)$ & 12.16 & 15.22 & 13.69 & 0.9965 & 7.28 \\
\hline Soluble nitrogen matter $(\%)$ & 0.0561 & 0.0098 & 0.0771 & 0.0174 & 22.57 \\
\hline Primary decomposition products of nitrogen matter (\%) & 0.0376 & 0.0735 & 0.0556 & 0.0147 & 26.53 \\
\hline Secondary decomposition products of nitrogen matter (\%) & 0.0185 & 0.0277 & 0.0231 & 0.0033 & 14.28 \\
\hline Coefficient of ripening $(\%)$ & 2.59 & 4.76 & 3.67 & 0.7206 & 19.62 \\
\hline Ash (\%) & 1.18 & 5.98 & 3.58 & 1.7575 & 49.09 \\
\hline $\mathrm{NaCl}(\%)$ & 0.79 & 4.80 & 2.80 & 1.5425 & 55.19 \\
\hline Acidity ( $\left.{ }^{0} \mathrm{SH}\right)$ & 66.72 & 75.26 & 70.99 & 2.7204 & 3.83 \\
\hline $\mathrm{pH}$ & 4.17 & 4.40 & 4.29 & 0.0870 & 2.03 \\
\hline \multirow{2}{*}{ Investigated parameters } & \multicolumn{5}{|c|}{ Statistical parameters } \\
\hline & $\min$. & max. & $X(n=6)$ & $\mathrm{S}_{\mathrm{d}}$ & $\mathrm{Cv}(\%)$ \\
\hline \multicolumn{6}{|c|}{ CHEESE MADE OF EWE'S MILK } \\
\hline TS (\%) & 46.72 & 57.16 & 51.94 & 3.6213 & 6.97 \\
\hline Moisture (\%) & 42.84 & 53.28 & 48.06 & 3.6213 & 7.54 \\
\hline MFFB (\%) & 63.00 & 72.99 & 67.99 & 3.0706 & 4.52 \\
\hline Fat $(\%)$ & 24.00 & 32.50 & 28.25 & 3.0236 & 10.70 \\
\hline FTS $(\%)$ & 49.06 & 59.02 & 54.04 & 3.3862 & 6.27 \\
\hline Total nitrogen $(\%)$ & 2.1947 & 2.9223 & 2.5585 & 0.2465 & 9.64 \\
\hline Proteins $(\%)$ & 14.00 & 18.64 & 16.32 & 1.5728 & 9.64 \\
\hline Soluble nitrogen matter $(\%)$ & 0.0841 & 0.1542 & 0.1192 & 0.0222 & 18.65 \\
\hline Primary decomposition products of nitrogen matter (\%) & 0.0564 & 0.1111 & 0.0838 & 0.0167 & 19.90 \\
\hline Secondary decomposition products of nitrogen matter (\%) & 0.0246 & 0.0431 & 0.0339 & 0.0063 & 18.73 \\
\hline Coefficient of ripening $(\%)$ & 3.67 & 6.93 & 5.30 & 1.1235 & 21.20 \\
\hline Ash (\%) & 1.77 & 6.34 & 4.06 & 1.6257 & 40.09 \\
\hline $\mathrm{NaCl}(\%)$ & 0.46 & 3.98 & 2.22 & 1.3319 & 60.00 \\
\hline Acidity $\left({ }^{0} \mathrm{SH}\right)$ & 19.40 & 90.77 & 55.09 & 27.7291 & 50.34 \\
\hline $\mathrm{pH}$ & 4.29 & 5.58 & 4.94 & 0.5376 & 10.89 \\
\hline
\end{tabular}


Based on results in table 1, according to cheese firmness cheese made of cow's milk belongs to group of soft cheeses with $69.40 \%$ MFFB. According to FTS content (47.44\%) it belongs to group of full fat cheeses with wide range of variety from $33.33 \%$ (full fat cheeses) to $61.55 \%$ (extra fat cheeses). According to cheese firmness, cheese made of ewe's milk belongs to group of soft cheeses with $67.99 \%$ MFFB. According to FTS content it belongs to group of full fat cheeses (54.04\%) (Codex alimentarius, 2000).

Table 2. Sensory evaluation of Svrljig white cheese in brine

\begin{tabular}{|c|c|c|c|c|c|c|c|c|c|}
\hline \multirow{2}{*}{$\begin{array}{c}\text { Number of } \\
\text { sample }\end{array}$} & \multirow{2}{*}{$\begin{array}{c}\text { Statistical } \\
\text { parameters }\end{array}$} & \multicolumn{6}{|c|}{ Investigated parameters } & \multicolumn{2}{|c|}{ Sensory evaluation } \\
\hline & & Appearance & Cross section & Colour & Aroma & Flavour & Consistency & $\mathrm{X}$ & $\%$ max. qual. \\
\hline \multicolumn{10}{|c|}{ CHEESE MADE OF COW'S MILK } \\
\hline \multirow{4}{*}{1} & $X(n=5)$ & 5.00 & 5.00 & 5.00 & 4.67 & 5.00 & 4.83 & \multirow{4}{*}{4.94} & \multirow{4}{*}{98.83} \\
\hline & $\mathrm{Sd}$ & 0.0000 & 0.0000 & 0.0000 & 0.2887 & 0.0000 & 0.2887 & & \\
\hline & $\mathrm{Cv}(\%)$ & 0.00 & 0.00 & 0.00 & 6.19 & 0.00 & 5.97 & & \\
\hline & $\% \max$ & 100.00 & 100.00 & 100.00 & 93.33 & 100.00 & 96.67 & & \\
\hline \multirow{4}{*}{2} & $X(n=5)$ & 4.83 & 4.50 & 5.00 & 4.83 & 4.67 & 4.67 & \multirow{4}{*}{4.71} & \multirow{4}{*}{94.17} \\
\hline & $\mathrm{Sd}$ & 0.2887 & 0.0000 & 0.0000 & 0.2887 & 0.2887 & 0.2887 & & \\
\hline & $\mathrm{Cv}(\%)$ & 5.97 & 0.00 & 0.00 & 5.97 & 6.19 & 6.19 & & \\
\hline & $\% \max$ & 96.67 & 90.00 & 100.00 & 96.67 & 93.33 & 93.33 & & \\
\hline \multirow{4}{*}{3} & $X(n=5)$ & 4.83 & 4.33 & 5.00 & 4.67 & 5.00 & 3.67 & \multirow{4}{*}{4.65} & \multirow{4}{*}{93.00} \\
\hline & $\mathrm{Sd}$ & 0.2887 & 0.2887 & 0.0000 & 0.5773 & 0.0000 & 0.2887 & & \\
\hline & $\mathrm{Cv}(\%)$ & 5.97 & 6.66 & 0.00 & 12.37 & 0.00 & 7.87 & & \\
\hline & $\% \max$ & 96.67 & 86.67 & 100.00 & 93.33 & 100.00 & 73.33 & & \\
\hline \multirow{4}{*}{4} & $X(n=5)$ & 5.00 & 4.83 & 5.00 & 4.67 & 4.17 & 5.00 & \multirow{4}{*}{4.61} & \\
\hline & $\mathrm{Sd}$ & 0.0000 & 0.2887 & 0.0000 & 0.2887 & 0.2887 & 0.0000 & & 9217 \\
\hline & $\mathrm{Cv}(\%)$ & 0.00 & 5.97 & 0.00 & 6.19 & 6.93 & 0.00 & & 32.1 \\
\hline & $\% \max$ & 100.00 & 96.67 & 100.00 & 93.33 & 83.33 & 100.00 & & \\
\hline & $X(n=5)$ & 4.17 & 4.17 & 4.83 & 4.67 & 4.50 & 4.00 & & \\
\hline 5 & $\mathrm{Sd}$ & 0.2887 & 0.2887 & 0.2887 & 0.2887 & 0.0000 & 0.0000 & & \\
\hline 5 & $\mathrm{Cv}(\%)$ & 6.93 & 6.93 & 5.97 & 6.19 & 0.00 & 0.00 & 4.39 & 87.83 \\
\hline & $\% \max$ & 83.33 & 83.33 & 96.67 & 93.33 & 90.00 & 80.00 & & \\
\hline & $X(n=5)$ & 4.33 & 4.67 & 4.83 & 4.33 & 4.17 & 4.33 & & \\
\hline 6 & $\mathrm{Sd}$ & 0.2887 & 0.2887 & 0.2887 & 0.2887 & 0.2887 & 0.2887 & & 8733 \\
\hline 6 & $\mathrm{Cv}(\%)$ & 6.66 & 6.19 & 5.97 & 6.66 & 6.93 & 6.66 & 4.31 & 87.33 \\
\hline & $\% \max$ & 86.67 & 93.33 & 96.67 & 86.67 & 83.33 & 86.67 & & \\
\hline & $X(n=5)$ & 3.83 & 4.50 & 4.83 & 4.17 & 3.83 & 4.83 & & \\
\hline 7 & $\mathrm{Sd}$ & 0.2887 & 0.5000 & 0.2887 & 0.2887 & 0.2887 & 0.2887 & 422 & 8433 \\
\hline 1 & $\mathrm{Cv}(\%)$ & 7.53 & 11.11 & 5.97 & 6.93 & 7.53 & 5.97 & 4.22 & 84.33 \\
\hline & $\% \max$ & 76.67 & 90.00 & 96.67 & 83.33 & 76.67 & 96.67 & & \\
\hline & & & CHEE & MADE & EWE'S & $\overline{\mathbf{L K}}$ & & & \\
\hline & $\bar{X}(\mathrm{n}=5)$ & 4.50 & 4.83 & 4.83 & 5.00 & 4.50 & 5.00 & & \\
\hline 1 & $\mathrm{Sd}$ & 0.5000 & 0.2887 & 0.2887 & 0.0000 & 0.0000 & 0.0000 & 71 & 0417 \\
\hline 1 & $\mathrm{Cv}(\%)$ & 11.11 & 5.97 & 5.97 & 0.00 & 0.00 & 0.00 & $4 . / 1$ & 94.17 \\
\hline & $\% \max$ & 90.00 & 96.67 & 96.67 & 100.00 & 90.00 & 100.00 & & \\
\hline & $X(n=5)$ & 4.83 & 4.83 & 5.00 & 4.50 & 4.50 & 4.50 & & \\
\hline 2 & $\mathrm{Sd}$ & 0.2887 & 0.2887 & 0.0000 & 0.5000 & 0.5000 & 0.0000 & 4.63 & 92.67 \\
\hline 2 & $\mathrm{Cv}(\%)$ & 5.97 & 5.97 & 0.00 & 11.11 & 11.11 & 0.00 & & \\
\hline & $\% \max$ & 96.67 & 96.67 & 100.00 & 90.00 & 90.00 & 90.00 & & \\
\hline & $X(n=5)$ & 5.00 & 5.00 & 5.00 & 4.17 & 4.17 & 4.83 & & \\
\hline 3 & $\mathrm{Sd}$ & 0.0000 & 0.0000 & 0.0000 & 0.5773 & 0.2887 & 0.2887 & 4.51 & 90.17 \\
\hline 3 & $\mathrm{Cv}(\%)$ & 0.00 & 0.00 & 0.00 & 13.86 & 6.93 & 5.97 & & \\
\hline & $\% \max$ & 100.00 & 100.00 & 100.00 & 83.33 & 83.33 & 96.67 & & \\
\hline & $X(n=5)$ & 3.50 & 4.50 & 4.33 & 3.33 & 4.33 & 4.17 & & \\
\hline 4 & $\mathrm{Sd}$ & 0.0000 & 0.0000 & 0.2887 & 1.1547 & 0.2887 & 0.2887 & 4.15 & 83.00 \\
\hline 4 & $\mathrm{Cv}(\%)$ & 0.00 & 0.00 & 6.66 & 34.64 & 6.66 & 6.93 & & \\
\hline & $\% \max$ & 70.00 & 90.00 & 86.67 & 66.67 & 86.67 & 83.33 & & \\
\hline & $X(n=5)$ & 4.83 & 4.00 & 5.00 & 4.17 & 4.00 & 3.50 & & \\
\hline 5 & $\mathrm{Sd}$ & 0.2887 & 0.0000 & 0.0000 & 0.7638 & 0.0000 & 0.5000 & 4.13 & 82.50 \\
\hline 5 & $\mathrm{Cv}(\%)$ & 5.97 & 0.00 & 0.00 & 18.33 & 0.00 & 14.29 & & \\
\hline & $\% \max$ & 96.67 & 80.00 & 100.00 & 83.33 & 80.00 & 70.00 & & \\
\hline & $X(n=5)$ & 4.33 & 3.83 & 4.67 & 4.00 & 4.00 & 4.17 & & \\
\hline 6 & $\mathrm{Sd}$ & 0.2887 & 0.7638 & 0.2887 & 0.0000 & 0.0000 & 0.2887 & 4.10 & 82.00 \\
\hline 6 & $\mathrm{Cv}(\%)$ & 6.66 & 19.92 & 6.19 & 0.00 & 0.00 & 6.93 & & \\
\hline & $\% \max$ & 86.67 & 76.67 & 93.33 & 80.00 & 80.00 & 83.33 & & \\
\hline
\end{tabular}


Cow's cheeses had a wide range of salt content $(0.79 \%-4.80 \%)$ with average $2.80 \%$. This wide range of salt content was present also in ewe's cheeses $(0.46 \%-3.98 \%)$, with average $2.22 \%$. It showed a big influence on ripening process, so coefficient of ripening of cow's cheese has been varied from $2.59 \%$ to 4.76 $\%$, with average $3.67 \%$. The ripening process in ewe's cheese was more intensive, with average coefficient of ripening $5.30 \%$.

Based on basic quality parameters, Svrljig cheese in brine is not much different than Zlatar, Sjenica or Homolje cheese (Dozet et al., 2000, 2002, Jovanović et al., 2004, 2005, Maćej et al., 2004a, 2005b, Savić et al., 2002).

Sensor evaluation includes the following characteristics: appearance, consistency, taste and aroma. Results are given in table 2

Among 7 white cow's cheese samples $57.14 \%$ had excellent value, and $42.86 \%$ were very good. The sample 1 was best evaluated, with $98.83 \%$ of maximal quality. Among 6 white ewe's cheese samples 50 $\%$ had excellent value, and 50\% were very good. The best evaluated sample had $94.17 \%$ of maximal quality. Evaluated cheeses had characteristic colour depended on used milk, closed curd with more or less holes and typical milky-acid taste and aroma.

View of Svrljig white cheese slice was illustrated in figure 1

\title{
Conclusion
}

From all the above the following can be concluded:

1. Svrljig white cheese is produced from ewe's milk, and white cheese in type of Svrljig cheese is produced of cow's or mixed cow's and ewe's milk. Both cheeses belong to a group of white cheeses in brine.

2. Both Svrljig white cheeses made of cow's and ewe's milk belong to group of soft cheeses according to curd firmness, with average moisture on a fat-free basis content $69.40 \%$ and $67.99 \%$ respectively.

3. According to fat in total solids content, cow's and ewe's milk cheeses belong to a group of full fat cheeses (47.44\% and 54.04\% respectively).

4. Both cheese varieties had acid curd ( $\mathrm{pH} 4.29$ and 4.94 respectively), which is specific for white cheeses in brine.

5. Evaluated cheeses had good sensor characteristics (taste, aroma, color and consistency) proper to milk kind (cows and ewes) from which were made.

6. Concerning quality and good sensor characteristics of Svrljig white cheese the detailed production research can be done. It can make the basis for geographic protection and protection of origin of this cheese.

\section{HEMIJSKE I SENZORNE KARAKTERISTIKE SVRLJIŠKOG BELOG SIRA}

\author{
Ognjen D. Maćej, Snežana T. Jovanović, Miroljub B. Barać, Sanja V. Seratlić, Tanja R. Vučić
}

Rezime: Cilj ovog rada je bio da se istaknu najvažnije karakteristike Svrljškog belog sira u salamuri. Svrljiški beli sir se izrađuje od ovčijeg, kravljeg ili mešanog kravljeg i ovčijeg mleka i odlikuje se visokim sadržajem masti u suvoj materiji $(47.44 \%$ i 54.04\%) i prema tom parametru kvaliteta spada u grupu punomasnih sireva. Prema sadržaju vode u bezmasnoj materiji sira pripada grupi mekih sireva $(69.40 \%$ i 67.99\%). Senzorne karakteristike sira bile su dobre, ukus, miris i boja bili su tipični za sireve izrađene od kravljeg i ovčijeg mleka. Visok stepen kiselosti i niska $\mathrm{pH}$ vrednost su karakteristični za grupu belih sireva u salamuri, u koju spada i Svrljiški sir.

Ključne reči: beli sirevi u salamuri, ovčije mleko, kravlje mleko 
References

1. CARIĆ, M., MILANOVIĆ, S., VUCELJA, D. (2000): Standardne metode analize mleka i mlečnih proizvoda. Prometej, Novi Sad.

2. CODEX ALIMENTARIUS (2000): Codex group standard for cheese in brine-codex stan 208-1999, FAO/WHO of UN, Rome.

3. DOZET, N., ADŽIĆ, N., STANIŠIĆ, M., ŽIVIĆ, N. (1996): Autohtoni mlječni proizvodi. Poljoprivredni institut, Podgorica, Silmir, Beograd

4. DOZET, N., MAĆEJ, O., JOVANOVIĆ, S. (2000): Proizvodnje mliječnih prerađevina u uslovima brdsko-planinskog područja. Prehrambena industrija-Mleko i mlečni proizvodi, 11 (3-4), 41-44.

5. DOZET, N., MAĆEJ, O., JOVANOVIĆ, S. (2002): Mogućnosti brdsko-planinskog područja za proizvodnju mliječnih proizvoda sa posebnim osvrtom na ovčije mljekarstvo. Biotehnologija u stočarstvu. Beograd, 127-135.

6. JOVANOVIĆ, S., BARAĆ, M., MAĆEJ, O. (2004): Karakteristike autohtone proizvodnje Homoljskog belog sira u salamuri. Biotehnologija u stočarstvu 20 (5-6), 157-163.

7. JOVANOVIĆ, S., MAĆEJ, O.,VUČIĆ, T., SERATLIĆ, S. (2005): Karakteristike autohtone proizvodnje Zlatarskog sira. Zbornik radova Simpozijuma "Mleko i proizvodi od mleka". Ur. Maćej, O., Jovanović, S. Tara, 84-86.

8. MAĆEJ, O., JOVANOVIĆ, S., DOZET, N., SERATLIĆ, S., VUČIĆ, T., SAVIĆ, Ž. (2004a): Tehnologija autohtone izrade Sjeničkog sira na širem području Sjeničko-pešterske visoravni. Mlekarstvo 3 (27), 931-933.

9. MAĆEJ, O., JOVANOVIĆ, S., BARAĆ, M. (2004b): Uticaj vrste mleka na kvalitet Sjeničkog sira i sireva u tipu Sjeničkog sira izrađenih autohtonom tehnologijom. Biotehnologija u stočarstvu, vol. 20(12), 109-117.

10. PEJIĆ, O., ĐORĐEVIĆ, J. (1963): Mlekarski praktikum. Naučna knjiga, Beograd.

11. RADOVANOVIĆ, R., POPOV-RALJIĆ, J. (2001): Senzorna analiza prehrambenih proizvoda. Poljoprivredni fakultet, Beograd i Tehnološki fakultet, Novi Sad.

12. SAVIĆ, Ž., DOZET, N., JOVANOVIĆ, S., MAĆEJ, O. (2002): Poboljšanje proizvodnje i kvaliteta Sjeničkog sira. Prehrambena industrija- Mleko i mlečni proizvodi, 13 (1-2), 44-50.

Figure 1. View of Svrljig white cheese slice

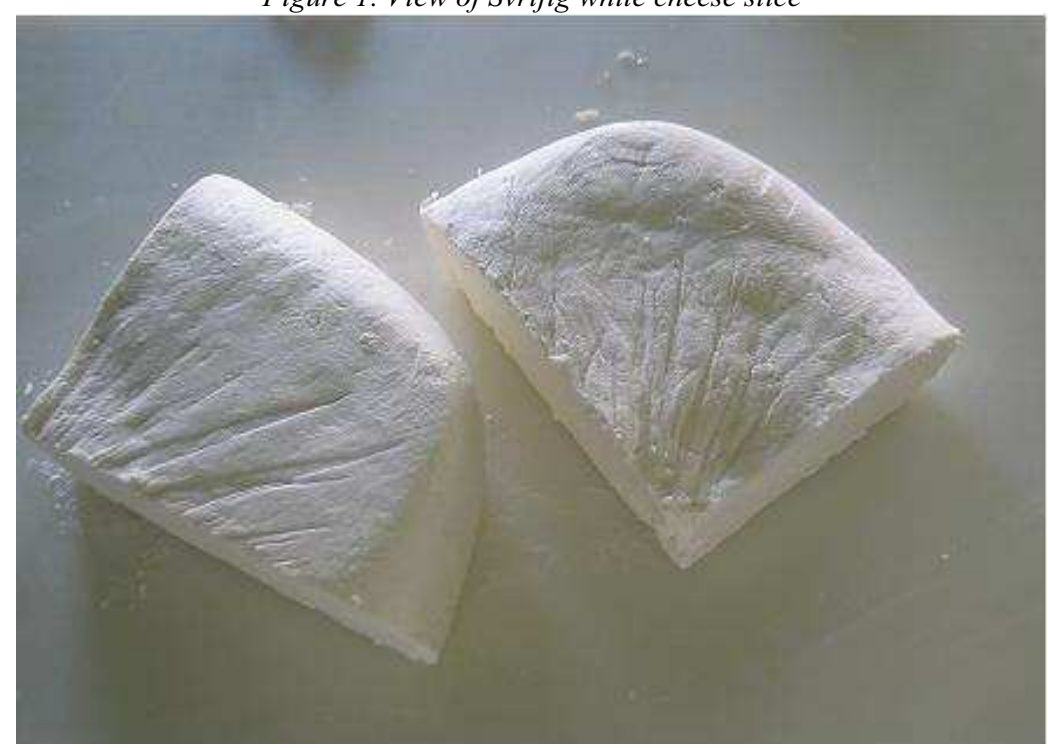

